\title{
Low-cost Antenna Positioning System Designed with Axiomatic Design
}

\author{
Joseph Timothy Foley ${ }^{1}{ }^{\star}$, Vladimir Omelianov ${ }^{1}$, Slawomir Koziel ${ }^{1}$, Adrian Bekasiewicz ${ }^{1}$ \\ ${ }^{1}$ Reykjavik University, Engineering Department, Menntavegur 1, 101 Reykjavik, Iceland
}

\begin{abstract}
The Engineering Optimization and Modeling Center at Reykjavik University has been carrying out research on antenna $\mathrm{CAD}$, including the simulation-driven design of novel antenna topologies. However, simulation is not enough to validate a design: a custom RF anechoic chamber has been built to quantify antenna performance, particularly in terms of field properties such as radiation patterns. Such experiments require careful positioning of the antenna in the chamber accurately in 3-axis with a short development time, challenging material constraints, and minimal funding. Axiomatic Design Theory principles were applied to develop an automated 3-axis positioner system for a reference antenna and the antenna to be calibrated. Each axis can be individually controlled with a repeatability of 1 degree. This 3000 USD device can be fabricated using easily available components and rapid prototyping tools.
\end{abstract}

\section{Introduction}

For the last 50 years, ways of communicating have been evolving more progressively than ever. Every ten years there has been a breakthrough in speed and range of wireless transmissions. This progress creates new challenges in the design of antenna structures that need to satisfy stringent requirements concerning electrical performance but also geometry constraints, which is particularly important for hand-held and wearable systems, as well as shortrange communications such as in Internet of things applications. Increasing complexity of antenna systems makes automated EM-simulation-driven design more and more important. The Engineering Optimization and Modeling Center (EOMC) at Reykjavik University has been involved in research concerning novel and computationally efficient techniques for design optimization of antennas, mostly in the $\mathrm{GHz}$ range, as well as the design of novel antennas (especially in terms of reduced-size structures). The following articles have been published concerning this area by the Center:

- Fast multi-objective surrogate-assisted design of multi-parameter antenna structures through rotational design space reduction [1]

- Compact UWB monopole antenna for internet of things applications [2]

- Suppressing sidelobes of linear phased array of microstrip antennas with simulation-based optimization [3]

- Structure and design optimization of compact UWB slot antenna [4]

The EOMC uses CST, Matlab, self-developed algorithms to automatically generate geometries and simulates the field characteristics of conceptual antennas. The cur-

\footnotetext{
^e-mail: foley@ru.is
}

rent simulation efforts have matured sufficiently that the next phase, validation, is needed. In short, the antennas need to be fabricated and tested for comparison to simulation results.

In 2015, the EOMC received funds from the Icelandic Center for Research (RANNIS) to build an anechoic chamber to prove their designs. A radio frequency (RF) anechoic chamber is a shielded room whose walls have been covered with a material that scatters or absorbs so much of the incident energy that it can simulate free space.

An example of a similar chamber at CSEM is shown in Fig. 1 and described thus:

The fully anechoic chamber with remote control offers a controlled propagation environment for antenna radiation pattern and gain measurement, e.g. for WPANs, BANs and UWB systems. The chamber consists of a metallic Faraday cage of $4.72 \times 4.05 \times 3.05 \mathrm{~m}$ with absorbent materials and a rotating platform on the inside. If required, the chamber can be made semi-anechoic by removing the absorbers on the floor. The useful space inside the chamber is $3.81 \times 3.14 \times 2.14 \mathrm{~m}$, and the chamber can be used for measurements above $400 \mathrm{MHz}$. The upper limit is determined by measurement equipment. The chamber is equipped with reference antennas, a $40 \mathrm{GHz}$ network analyzer, and a $26.5 \mathrm{GHz}$ spectrum analyzer. It can be used for 2-D antenna radiation pattern measurements, total radiated power (TRP) measurements and EMC measurements. A human body phantom and a human hand phantom are also available [5]. 


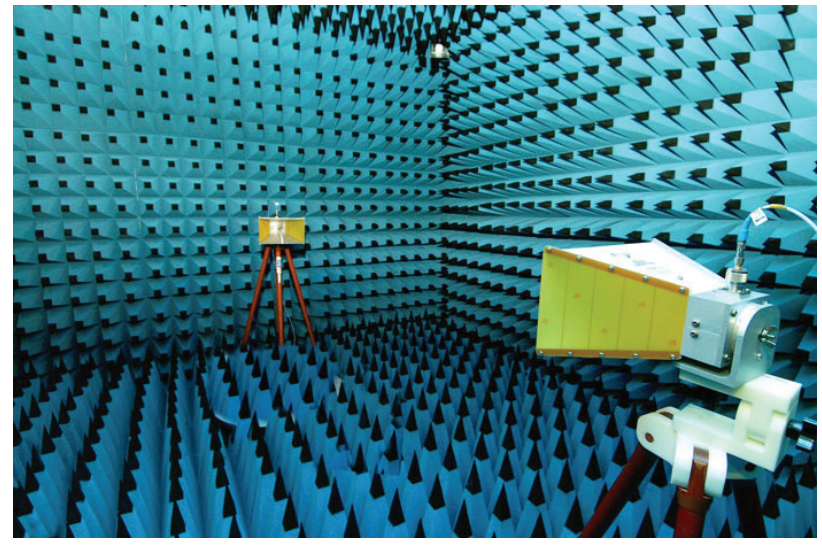

Fig. 1. Example of a fully equipped anechoic chamber at CSEM. Note the anechoic damper spikes, manual positioner, and wooden tripods [5].

As seen in the example, for a complete system, more is needed than just an RF chamber designed to eliminate interference and reflections. A mechanism is needed to position the antennas accurately and repeatably. The majority of the funds allocated were devoted to the purchase, configuration, and installation of the chamber; much smaller funds remained (3000 USD) for the positioning mechanism.

The main challenge with designing an appropriate positioner is that each anechoic chamber is used for very specific measurements: [6]:

- Radiation Pattern and Gain Measurement

- Phase Measurement

- Polarization Measurement

- Impedance Measurement

- Scale Model Measurement

- SAR (Specific Absorption Rate) Measurement

The size of the measured antennas and frequencies have a big role as well. As a result, testing many combinations of these variables requires versatile equipment.

\subsection{Prior Art}

Consider two commercially available positioner systems:

- TDK 3-Dimensional Manipulator, Model 3DM01LDs (Fig. 2a)

- Non-conductive commercial grade fiberglass and $\mathrm{ABS}$ plastic

-2 axis of rotation

- Fiber optic interface

- Accuracy: $\pm 2^{\circ}$ standard with $\pm 0.5^{\circ}$ accuracy

- Rotation Angle: $360^{\circ}$ continuous

- Price: 39000 USD (does not include control unit)

- Sunol EUT positioner, Model ELA275 (Fig. 2b)

- Continuous Rotation allowed in both Elevation \& Azimuth (with optional components)

- Low-Density EUT holder

- Low RF Cross-Section materials above drive units

- Portable (no permanent installation necessary)
- Remote Azimuth Drive Option separating drive units

- Fiber Optic connection to Controller (requires SC104V System Controller)

- Price: 31000 USD (with a control unit)

- Precision: $\pm 0.25^{\circ}$

- ETS-Lindgren 2120CR Multi-Axis Positioner (Fig. 2c)

- 360 Independent Rotation in Both Theta and Phi Axes, Continuous Rotation in Phi Axis

- Angular Positioner Accuracy Better than \pm $0.025^{\circ}$

- Price: 54000-80000 USD (depending on configuration and excluding controller)

As we can see all of these solutions have the following limitations, making them unsuitable for our situation:

- All of the towers do not have an axis of rotation at the same point

- Custom mounting plates required for each antenna design

- Installation requires a flat surface

- Absence of coaxial connection on the antennas mount as a default option

- The average price of the mentioned above manipulators is 40000 USD without a controller. This puts the positioners well outside the range of most small grants.

\section{Design}

This positioner design was developed using the Axiomatic Design methodology which focuses on the mapping between customer needs into instantiation [10]. The customer is the antenna measurement team and our top level need is:

$\mathbf{C N}_{0}$ Measure the performance of the antenna under test.

Other needs emerge decomposing this main goal:

$\mathbf{C N}_{1}$ Compatible with our anechoic chamber

$\mathbf{C N}_{2}$ Reference antennas of interest are up to $260 \times 260 \times$ $260 \mathrm{~mm}$

$\mathbf{C N}_{3}$ Designed antennas are $10 \times 10 \times 2 \mathrm{~mm}$ to $300 \times 30 \times$ $2 \mathrm{~mm}$

$\mathbf{C N}_{4}$ Do not exceed budget of 3000 USD

$\mathbf{C N}_{5}$ Can quickly change designed antennas

$\mathbf{C N}_{6}$ Easily reconfigured for different kinds of experiments

$\mathbf{C N}_{7}$ Re-orient the antenna without human intervention

$\mathbf{C N}_{7}$ Complete design to implementation in 3 months

Customer Needs are translated into Constraints (Cs) and Functional Requirements (FRs) which are fulfilled by Design Parameters (DPs). Constraints are designed envelopes that affect many elements within the system but do not define its functionality.

\subsection{Constraints}

$\mathbf{C}_{1}$ Each module mass below $20 \mathrm{~kg}$

$\mathbf{C}_{2}$ Use coax cables of fixed lengths 100, 200, 400, 600, $800 \mathrm{~mm}$ 


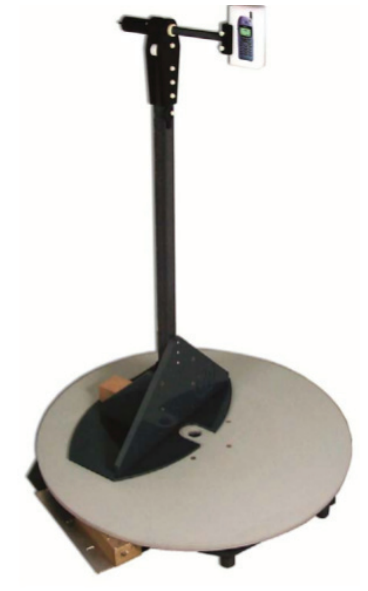

(a) TDK Model 3DM01LDs [7].

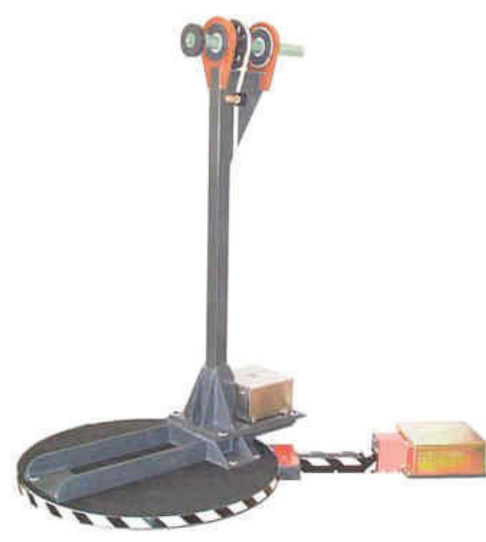

(b) Sunol Model ELAZ75 [8].

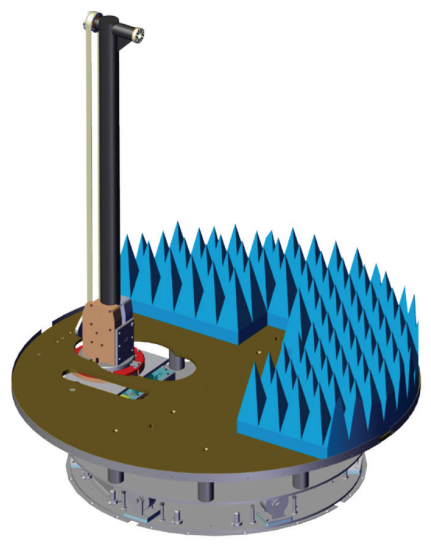

(c) ETS-Lindgren Model 2120CR [9].

Fig. 2. Existing commercial 3D positioners for use in RF anechoic chambers.

$\mathbf{C}_{3}$ Have time that required to install measuring antennas be at most 5 minutes

$\mathbf{C}_{4}$ Have easy access to cables and connectors

$\mathbf{C}_{5}$ Have high repeatability of measurements

$\mathbf{C}_{6}$ Time constraint: 3 months

$\mathbf{C}_{7}$ Fabrication using equipment at Reykjavik University

$\mathbf{C}_{8}$ Support reference antennas with weight up to $3 \mathrm{~kg}$

$\mathbf{C}_{9}$ Support reference antennas with dimensions up to $260 \times 260 \times 260 \mathrm{~mm}$

$\mathbf{C}_{10}$ Support dimensions of measured antennas that are $10 \times 10 \times 2 \mathrm{~mm}$ to $300 \times 30 \times 2 \mathrm{~mm}$

$\mathbf{C}_{11}$ Keep cables from bending over $10 \mathrm{~mm}$ radius

$\mathbf{C}_{12}$ Do not exceed budget of 3000 USD

\subsection{Functional Requirements (FRs)}

Best practices for FRs are that they must start with an action or transformative verb and able to be validated. [10, 11] The CNs are considered to generate top-level Functional Requirements (FRs):

$\mathbf{F R}_{0}$ Rotate measured antenna in two axes and reference antenna in polarization angle without introducing interfering elements

$\mathbf{D P}_{0}$ Stepper-driven 2-axis positioner for measured antenna and 1-axis positioner for reference antenna both made primarily of plastic

For brevity, we have combined the results of zigzagging into the following FR and DP decompositions.

$\mathbf{F R}_{1}$ Rotate measured antenna in two axes with 1-degree resolution.

$\mathbf{F R}_{1.1}$ Rotate measured antenna in $\mathrm{XZ}$ (vertical) plane by $180^{\circ}$.

$\mathbf{F R}_{1.2}$ Rotate measured antenna in XY (horizontal) plane by $360^{\circ}$.

$\mathbf{F R}_{1.3}$ Mount measured antenna with $1 \mathrm{~mm}$ repeatability without tools.

$\mathbf{F R}_{2}$ Measure affect of reference antenna polarization angle with $1^{\circ}$ resolution.

$\mathbf{F R}_{2.1}$ Rotate reference antenna XZ (vertical) plane by $180^{\circ}$.
$\mathbf{F R}_{2.2}$ Fixture multiple commercial reference antenna mounting schemes.

$\mathbf{F R}_{2.3}$ Align relative position of the measured and reference antennas.

$\mathbf{F R}_{3}$ Avoid introducing additional RF interference during antenna excitation

$\mathbf{F R}_{3.1}$ De-energize motor controllers during measurement.

$\mathbf{F R}_{3.2}$ Supply signal to antennas.

$\mathbf{F R}_{3.3}$ Supply power and control signals in shielded cables to components.

$\mathbf{F R}_{3.4}$ Eliminate any material that would change antenna performance.

$\mathbf{F R}_{4}$ Provide interface for user to setup and run experiment.

$\mathbf{F R}_{5}$ Stand on the chamber's spiked floor.

\subsection{Design Parameters}

Design Parameters are the methods, elements, and quantities that allow for instantiation. To best describe such parameters, they must start with a noun and be something that can be compared or quantified $[10,11]$.

$\mathbf{D P}_{1}$ Stepper motor and timing belt driven "receiver tower" to operate with measured (small) antennas

$\mathbf{D P}_{1.1}$ Plastic vertical rotary disk on wheels

$\mathbf{D P}_{1.2}$ Plastic horizontal rotary plate on custom nylon-ball thrust bearing

$\mathbf{D P}_{1.3}$ Plastic adjustable small antenna holder with hand-tightened clamp to SMA connector on antenna

$\mathbf{D P}_{2}$ Stepper motor and timing belt driven "transmitter tower" to operate with reference (big) antennas

$\mathbf{D P}_{2.1}$ Plastic vertical rotor on horizontal shaft with ball bearings

$\mathbf{D P}_{2.2}$ Plastic antenna adapter plate with slots in most common antenna mounting configurations

$\mathbf{D P}_{2.3}$ Detachable laser inclinometer located in front of axis of rotation 
$\mathbf{D P}_{3}$ Interference mitigation procedures and elements including RF absorbing foam

$\mathbf{D P}_{3.1}$ Smoothie Motor controller able to deenergize motors during operation (Controller device)

DP $_{3.2}$ Flexible coax cable and rotating couplings

DP $_{3.3}$ DIN9 shielded cables

$\mathbf{D P}_{3.4}$ No plastic in axis of measurement, metal components $100 \mathrm{~mm}$ from surface of measured antenna

DP $_{4}$ MATLAB Software interface between network analyzer and motor controller

DP $_{5}$ Aluminum Tripod with $1 / 4$ " connector covered in RF absorbent foam

\subsection{Design Matrix}

This leads to creation of a design matrix from the Cartesian product of coupling coefficients on FR and DP vectors (Eq. 1) $[10,11]$. In many cases the coupling quantitative value is unknown (or hard to quantify) so first-order effects are denoted with the variable $X$.

$$
\left\{\begin{array}{l}
\mathrm{FR}_{1} \\
\mathrm{FR}_{2} \\
\mathrm{FR}_{3} \\
\mathrm{FR}_{4} \\
\mathrm{FR}_{5}
\end{array}\right\}=\left[\begin{array}{ccccc}
X & 0 & 0 & 0 & 0 \\
0 & X & 0 & 0 & 0 \\
X & X & X & 0 & 0 \\
0 & 0 & 0 & X & 0 \\
0 & 0 & 0 & 0 & X
\end{array}\right]\left\{\begin{array}{l}
\mathrm{DP}_{1} \\
\mathrm{DP}_{2} \\
\mathrm{DP}_{3} \\
\mathrm{DP}_{4} \\
\mathrm{DP}_{5}
\end{array}\right\}
$$

This design matrix is decoupled i.e. path-dependent, so the order in which things are designed is important. It should be not surprising that $\mathrm{FR}_{3}$ is coupled to $\mathrm{FR}_{1}$ and $\mathrm{FR}_{2}$ due to the concern of RF interference during design. The design matrix decompositions for $\mathrm{FR}_{1}$ and $\mathrm{FR}_{2}$ are uncoupled (diagonal) due to the modularity of the design and are omitted. The second level design matrix in Eq. 2 is decoupled, due to the in-axis interference affecting other decisions.

$$
\left\{\begin{array}{l}
\mathrm{FR}_{3.1} \\
\mathrm{FR}_{3.2} \\
\mathrm{FR}_{3.3} \\
\mathrm{FR}_{3.4}
\end{array}\right\}=\left[\begin{array}{cccc}
X & 0 & 0 & 0 \\
0 & X & 0 & 0 \\
0 & X & X & 0 \\
0 & X & X & X
\end{array}\right]\left\{\begin{array}{l}
\mathrm{DP}_{3.1} \\
\mathrm{DP}_{3.2} \\
\mathrm{DP}_{3.3} \\
\mathrm{DP}_{3.4}
\end{array}\right\}
$$

PTC CREO Parametric 3.0 was used to develop the CAD model based upon these DPs.

\subsection{Receiver tower}

The biggest challenges in this design are avoiding metal elements, large variation in antennas, the routing of cables, and having no obstacles in front and behind of the antennas. This is why the circular part of the tower persisted through the stages of the design. The early version in Fig. 3 had the antenna wire wrapping around the wheel created an unacceptably large blind spot in measurements.

In the next version, the wheel was rotated at 180 degrees allowing for easier cable management. However, the weight of tower, considering it was made from $10 \mathrm{~mm}$ Plexiglas was at $24 \mathrm{~kg}$, violating $\mathrm{C}_{1}$. By "reinventing" the

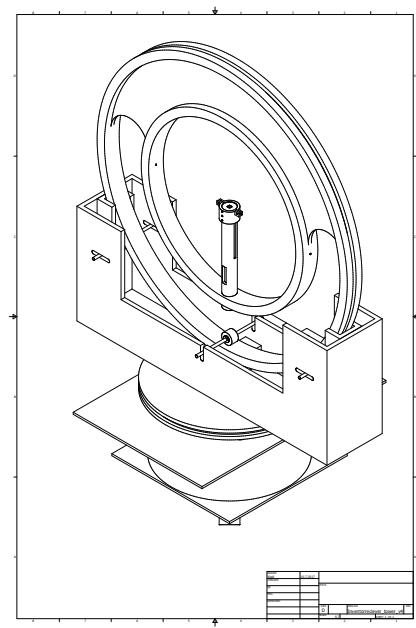

Fig. 3. Early design of the receiver tower (V4).

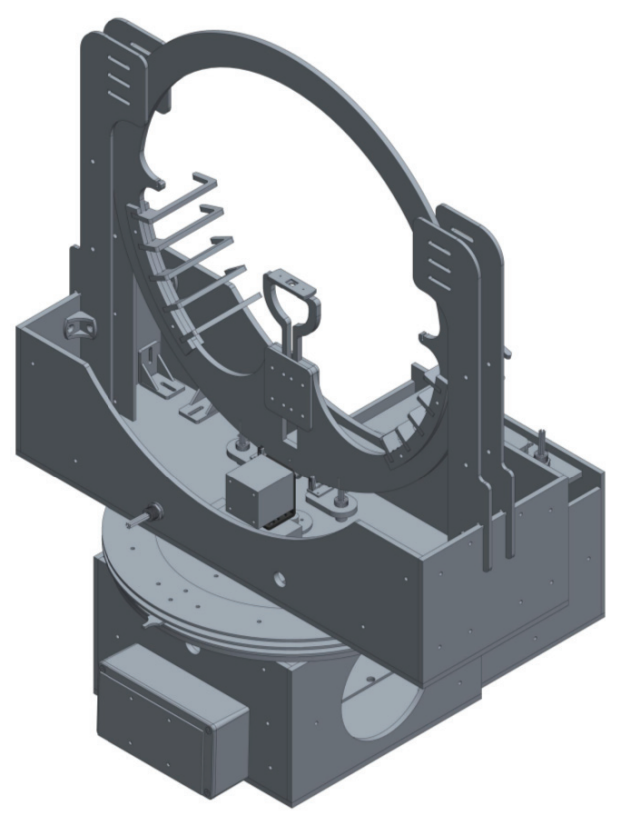

Fig. 4. Design of the receiver tower at its final stage (V5.3). Note the cable guide fingers on the side of the ring.

wheel into a socket that will hold the antenna holder, the design resulted in $10 \mathrm{~kg}$ device after reducing the width of Plexiglas to $6 \mathrm{~mm}$ as shown in Fig. 4. A cutaway is shown in Fig. 5 to show the main components.

The mass of the tower is approximately $10 \mathrm{~kg}$ (including the antenna) and total material cost 1500 USD (including cables).

Rotating elements are driven by timing belt connected to a NEMA 23 bipolar hybrid stepper motor with 200 steps per revolution $\left(N_{\text {stepper }}=200\right)$. Each stepper motor has a sprocket for connecting to the belt with $N_{\text {sprocket }}=20$ teeth. In the case of the large vertical ring, the pulley of the stepper drives an inverted timing belt adhered to the side of the ring with $N_{\text {wheel } 1}=738$ teeth. The horizontal platform rolls on a custom made plastic ball thrust bearing 


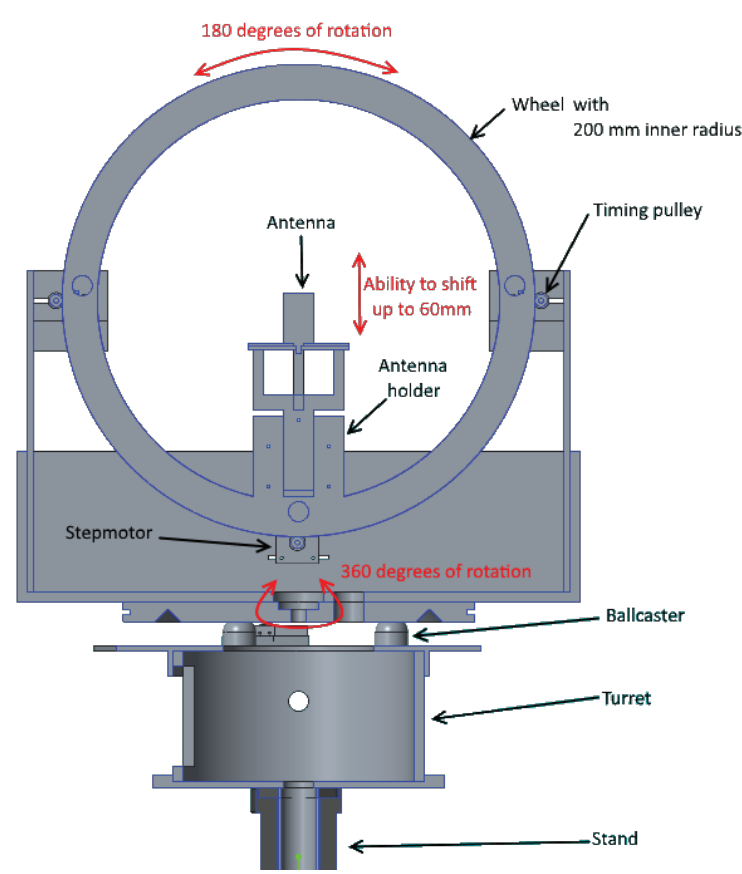

Fig. 5. Annotated cut-away drawing of the receiver tower.

with a timing belt of $N_{\text {wheel } 2}=533$ teeth. To calculate the angular resolution $\left(\theta_{R}\right)$, the timing belt gear ratio must be taking into account.

$$
\begin{aligned}
\theta_{\text {step }} & =\frac{360^{\circ}}{N_{\text {step }}} \\
R_{\text {wheel }} & =\frac{N_{\text {wheel }}}{N_{\text {sprocket }}}
\end{aligned}
$$

where

The resolution for a given step is proportional to the wheel's ratio; combining Eq. $3 \& 4$ results in the angular resolution $\left(\theta_{R}\right)$ :

$$
\theta_{R}=\frac{\theta_{\text {step }}}{R_{\text {wheel }}}=360^{\circ} \frac{N_{\text {sprocket }}}{N_{\text {step }} N_{\text {wheel }}}
$$

The vertical ring has a resolution of $0.049^{\circ}$ per step. The horizontal rotation has a resolution of $0.068^{\circ}$ per step. Both are well within the requested resolution of $1^{\circ}$.

\subsection{Transmitter tower}

The transmitter tower has a much simpler design due to fewer movement axis. The most challenging design problem is that reference antennas varying greatly in shape and weight. The current design is shown on Fig. 10 on page 7 . This stepper motor and timing belt configuration has $N_{\text {wheel3 }}=130$ resulting in a resolution of $0.277^{\circ}$ per step, which meets the requirements.

It is worth noting that the center of mass shifts depending on the type of antennas attached in this design. To connect the antennas an adapter plate is laser-cut for each antenna. The weight of the device is approximately $15 \mathrm{~kg}$ (including the antenna) and material cost 800 USD

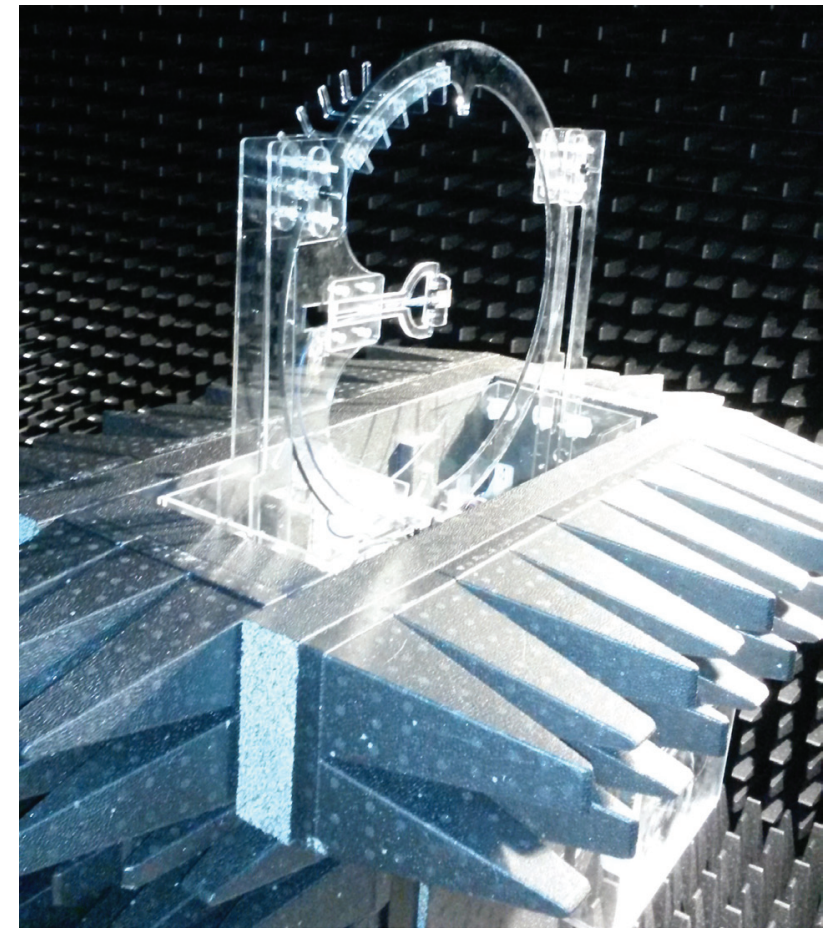

Fig. 6. 3-D configuration of the receiver tower.

(including cables) According to customer restrictions, the proximity of metals doesn't heavily affect reference antenna performance as long as it is off axis.

\subsection{Control unit}

The control unit is built around a SmoothieBoard 500 USD Open Source Hardware CNC controller board based on the LPC1768 Cortex-M3 chip [12]. Interfacing with the controller is done through GCODE over a USB-serial connection. Our Matlab program commands the controller to move then de-energizes the motors after the motion to reduce the noise. In addition, Matlab 2015 added support of VISA protocol for direct control of our Anritsu MS4640A network analyzer.

\section{Results and Discussion}

The effect of the mechanical components and fixtures of the receiver tower (Fig. 6) on the accuracy of the measured field characteristics of the antenna at hand has been validated.

More specifically, a comparison between simulation and measurement data has been performed for several test designs. Despite utilization of low-permittivity plastic as the construction material of the tower, rotation of the ring in the vicinity of the line of sight between the transmitter and receiver tower results in strong distortions of the measured signal. Moreover, an additional set of tests has indicated that the base of the rotary tower, even though entirely covered by absorbing material, noticeably influences the characteristics of the antenna under test due to varying 


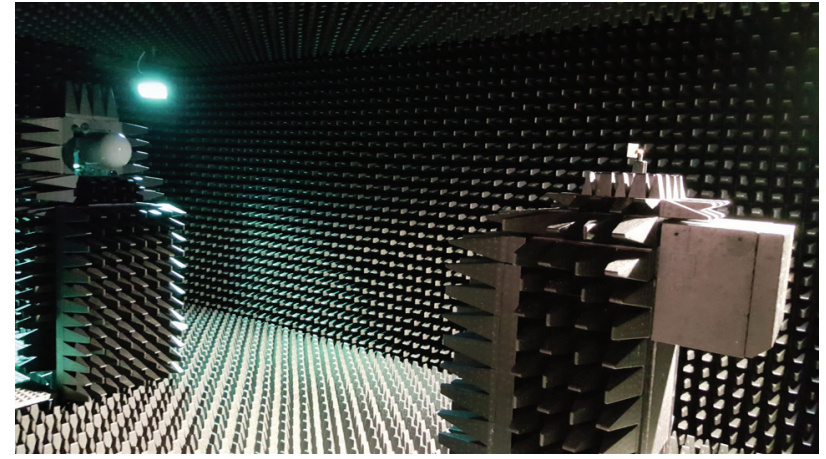

Fig. 7. Reykjavik University's RF anechoic chamber with the two towers installed. Receiver tower is in 2-D configuration.

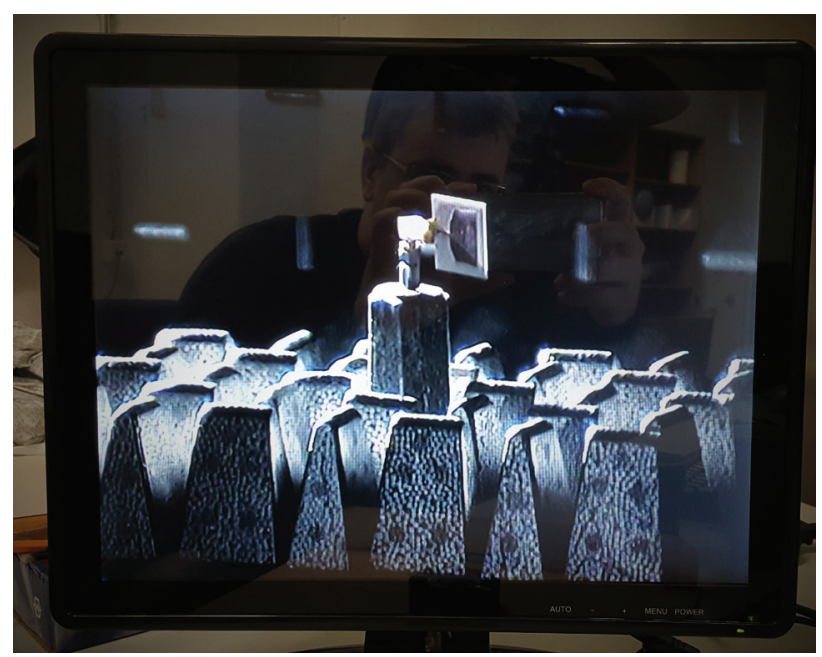

Fig. 8. 2-D measurements in progress on monopole antenna as viewed through the test chamber CCTV system.

patterns of signal reflections from the rotating absorber tapers.

The aforementioned effects could be compensated for by appropriate post-processing of the measured signal. For example, distortions caused by rotations of the receiver tower could be simulated and then subtracted from the measured signal. However, the major problem is that accurate simulations of tower-induced distortions can be performed only using computationally expensive full-wave EM model, evaluation of which is a matter of days per simulation. At the same time, for correction of 3-D measurement data, at least a few hundred of EM simulations of the tower at different positions are required. Clearly, such a procedure is numerically prohibitive.

Notwithstanding, about $90 \%$ of antenna measurements require only horizontal rotations of the receiver antenna. From this perspective, simplification of the tower construction seems to be a reasonable solution. Due to the modular design, reconfiguration was accomplished without significant changes to the system (cf. Fig. 7).

A designed monopole antenna under test mounted on a reconfigured receiver tower is shown in Fig. 8.

The measurements in the E- and H-planes took approximately 3 minutes each $\left(360^{\circ}\right.$ rotation with a step size of $5^{\circ}$ ). It should be noted that 500 frequency points in a range from $1 \mathrm{GHz}$ to $20 \mathrm{GHz}$ are captured at a time for each allocation of the antenna. Consequently, no extra calibration of the system is required for varying frequency ranges. Fig. 9 on page 7 shows a comparison of simulated and measured radiation pattern characteristics of the antenna at selected frequencies. Using Axiomatic design a complete RF antenna positioning system has been developed, designed and built, with an overall budget of 3000 USD.

\section{References}

[1] S. Koziel, A. Bekasiewicz, Fast multi-objective surrogate-assisted design of multi-parameter antenna structures through rotational design space reduction, IET Microwaves, Antennas \& Propagation (2016)

[2] A. Bekasiewicz, S. Koziel, Compact UWB monopole antenna for Internet of Things applications, Electronics Letters (2016)

[3] S. Koziel, S. Ogurtsov, A. Bekasiewicz, Suppressing sidelobes of linear phased array of microstrip antennas with simulation-based optimization, Metrology and Measurement Systems (2016)

[4] A. Bekasiewicz, S. Koziel, Structure and design optimisation of compact UWB slot antenna, Electronics Letters (2016)

[5] G. Dash, How RF Anechoic Chambers Work, Glen R. Dash Charitable Foundation (2005)

[6] Antenna measurements (2011), http: //www . antenna-theory.com/measurements / antenna.php

[7] Antenna tripods, masts and positioners - EUT manipulators - horn positioners (c2001-2016), http: //www . ramayes.com/tripods_masts_ positioners.htm

[8] Sunol sciences corporate website (2015), http:// www. sunolsciences.com/

[9] 2120cr multi-axis positioner (maps) all info (2014), http://www . ets-lindgren. com/all/2120CR

[10] N.P. Suh, The Principles of Design (Oxford University Press, 1990)

[11] N.P. Suh, D.S. Cochran, P.C. Lima, Manufacturing System Design, in 48th General Assembly of CIRP, Annals of the CIRP (1998), Vol. 47, pp. 627-639

[12] SmoothieBoard: Open Source Hardware CNC controller board (2015), http: //smoothieware.org 


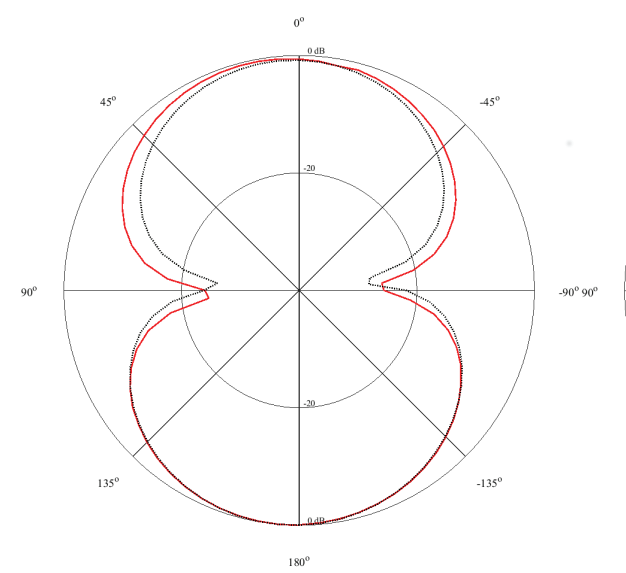

(a) $2 \mathrm{GHz}$ on the E plane

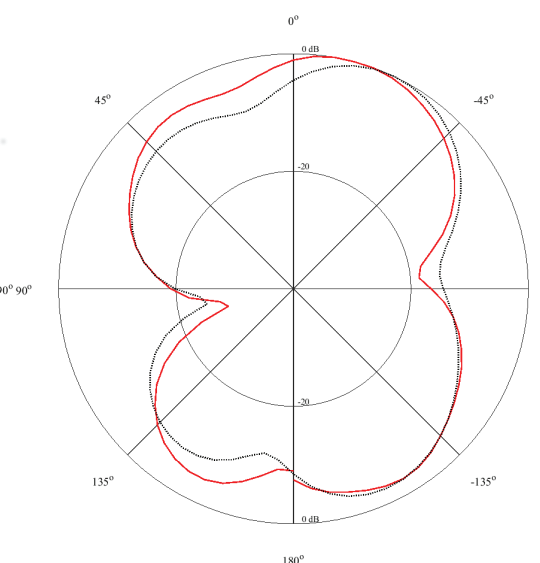

(b) $9 \mathrm{GHz}$ on the E plane

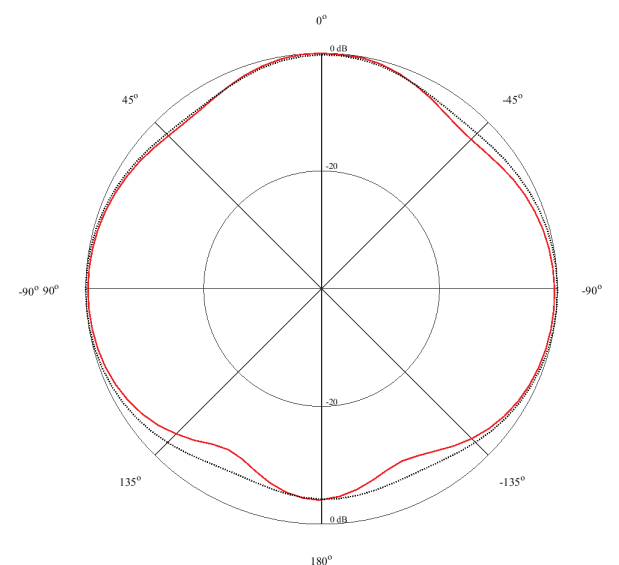

(c) $9 \mathrm{GHz}$ on the H plane

Fig. 9. Simulated (dotted black) and measured (red) radiation pattern characteristics of the monopole antenna of Fig. 8.
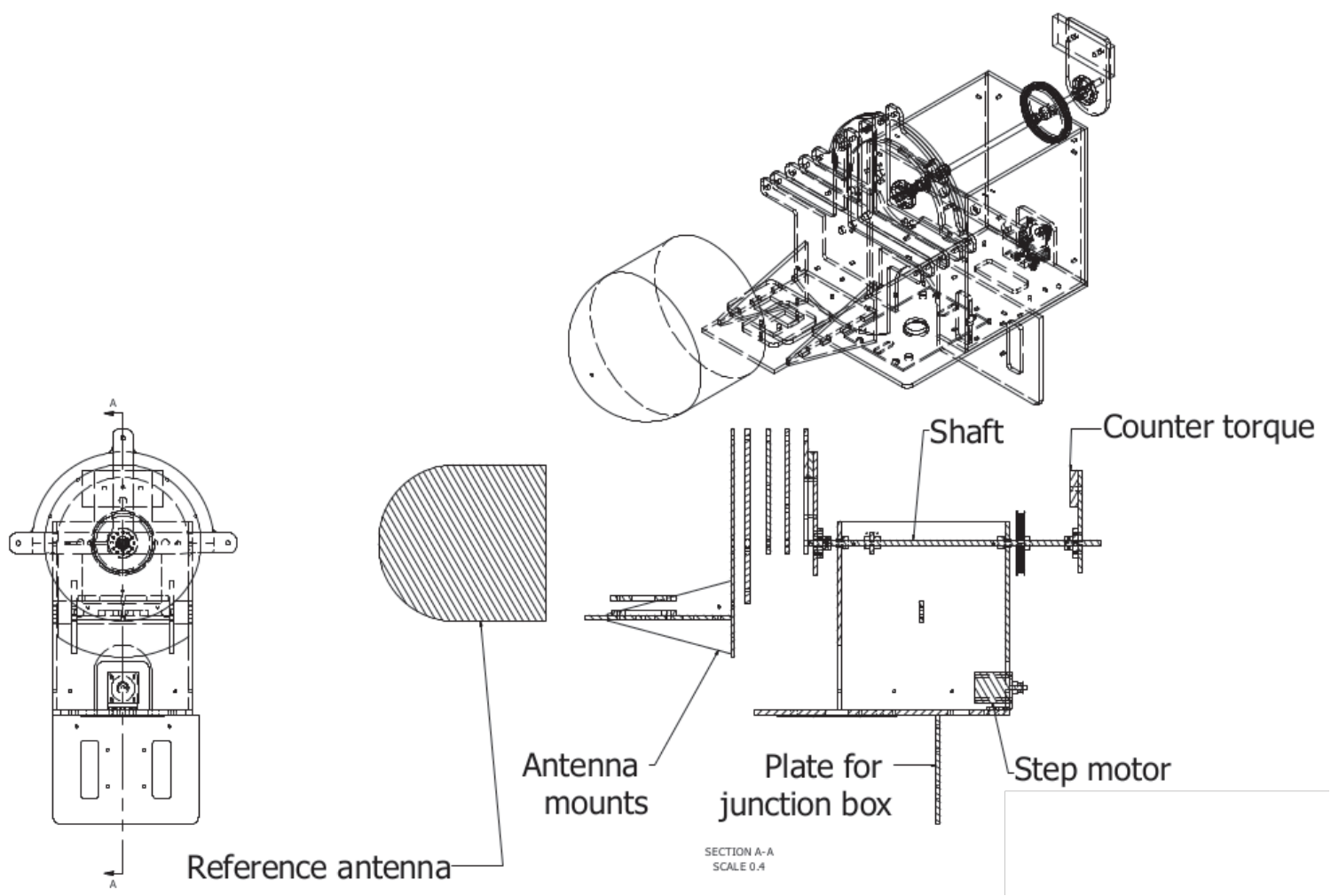

Fig. 10. The transmitter tower assembly must be able to securely mount a large variety of reference antenna geometries and masses. 